\title{
Análise dos Fatores Críticos para o Sucesso de Cursos de Graduação em Computação: Um Estudo de Caso na Região Amazônica
}

\author{
Marisa C. M. A. de Andrade ${ }^{1}$, Nelson C. Sampaio Neto ${ }^{1}$, Decíola F. de Sousa ${ }^{2}$, \\ Rafael O. Chaves ${ }^{3}$, Gláucio H. S. de Carvalho ${ }^{1}$ \\ ${ }^{1}$ Programa de Pós-Graduação em Ciência da Computação - PPGCC - Universidade \\ Federal do Pará (UFPA) - Caixa Postal 479 - 66.075-110. Belém-PA-Brasil \\ ${ }^{2}$ Instituto Ciberespacial - Universidade Federal Rural da Amazônia (UFRA) \\ Av. Presidente Tancredo Neves, 2501. 66077-901. Belém-PA-Brasil \\ ${ }^{3}$ Faculdade de Engenharia da Computação e Telecomunicações - Universidade Federal \\ do Pará (UFPA) - Caixa Postal 479 - 66.075-110. Belém-PA-Brasil

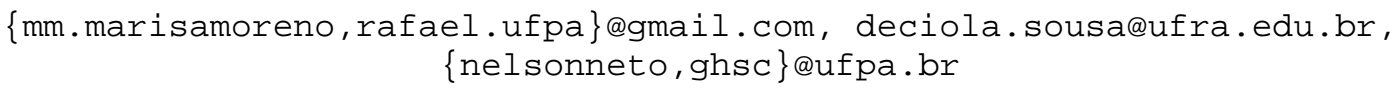

\begin{abstract}
The purpose of this article is to identify the Critical Success Factors (CSF) undergraduate courses in Computer from the viewpoint of students. FCS were searched and grouped into four categories: Institution, Teacher, Student and Institution Support Services. Each category consists of several indicators. We used the five point Likert questionnaires to generate quantitative descriptions. The participants were 284 students from two federal universities in the Amazon. The results were determined by statistical analysis of the Likert items in each category. The consistency of the questionnaire was 0.975 according the Cronbach's alpha coefficient. The results showed that the most important was the FCS Teacher.
\end{abstract}

Resumo. O objetivo deste artigo é identificar os Fatores Críticos de Sucesso (FCS) de cursos de graduação em Computação na ótica dos alunos. Os FCS foram pesquisados e agrupados em quatro categorias: Instituição, Docente, Aluno e Serviços de Apoio da Instituição. Cada categoria é composta de vários indicadores. Utilizou-se o questionário Likert de cinco pontos para gerar descrições quantitativas. Participaram da pesquisa 284 alunos de duas universidades federais da Amazônia. Os resultados foram determinados pela análise estatística dos itens Likert em cada categoria. A confiabilidade do questionário foi de 0,975 segundo o coeficiente alpha de Cronbach. Os resultados apontaram que o FCS mais relevante foi o FCS Docente.

\section{Introdução}

A forte demanda da sociedade por profissionais qualificados na área da Computação vem ao encontro do crescimento na oferta de novos cursos nesta área. Nunes (2012), membro da Sociedade Brasileira de Computação (SBC), utilizou dados fornecidos pelo Instituto Nacional de Estudos e Pesquisas Educacionais Anísio Teixeira (INEP), vinculado ao Ministério da Educação (MEC), para apontar um aumento contínuo do quantitativo de cursos de Educação Superior em Computação no país. 
Os dados revelam que, em 2005, existiam 1.690 cursos superiores da área da Computação; e, em 2012, existiam 2.231 cursos, indicando um aumento de $32 \%$ no quantitativo de cursos oferecidos pelas IES em um período de sete anos. Este cenário sugere competitividade entre as IES nacionais, tornando oportuna a realização do monitoramento contínuo de suas estratégias que auxiliem na tomada de decisões, por parte das instituições, a fim de identificar meios de atrair, reter seus estudantes e ainda apontar aspectos importantes para a obtenção de sucesso.

Uma alternativa para auxiliar os processos de tomada de decisões é a abordagem teórica dos Fatores Críticos de Sucesso (FCS), proposta por Rockart (1979). O autor definiu FCS como sendo "um número limitado de áreas nas quais os resultados, se forem satisfatórios, irão assegurar um desempenho competitivo e próspero para a organização".

Os FCS já foram adaptados para a área acadêmica e educacional (Retamal, 2009) e são usados por diversos pesquisadores para auxiliar gestores no processo de gestão da IES, sendo encontrada uma vasta seleção de trabalhos na literatura nacional e internacional sobre a utilização dos FCS na Educação (Oliveira, Santos e Kalatzis, 2007). Entretanto, desconhece-se registros na literatura sobre a identificação e análise dos FCS aplicados à cursos de graduação em Computação.

Diante da relevância da técnica dos FCS na área da Educação e da importância da avaliação por parte dos alunos dos cursos oferecidos pelas IES, o objetivo principal deste trabalho é identificar FCS para cursos de graduação em Computação e discutir os resultados destes baseados nos dados coletados na aplicação de questionários para alunos dos cursos de Computação da Universidade Federal Rural da Amazônia (UFRA) e da Universidade Federal do Pará (UFPA), ambas situadas na Região Amazônica. Nesse sentido, foi realizada uma análise estatística de indicativos de desempenho para dar suporte à tomada de decisões no contexto educacional regional.

O artigo está organizado de tal modo que na Seção 2 é apresentado o referencial teórico. A metodologia utilizada para identificação dos FCS é mostrada na Seção 3. A apresentação e análise dos dados são mostradas na Seção 4 e nas Seções 5 e 6 são apresentados os resultados e as considerações finais, respectivamente.

\section{Referencial teórico}

Segundo Rockart (1979) os FCS são as poucas áreas-chave em que o bom desempenho assume um papel importante para que a missão da organização seja alcançada, mas que, caso o desempenho não ocorra como previsto, os resultados da organização tendem a serem inferiores do que havia sido planejado.

Os FCS são utilizados em diversas áreas e determinam o sucesso e o fracasso da organização em termos de efetividade (Júnior; Polizel; Silva, 2012). Assim, reconhecese a relevância dos FCS como um importante mecanismo que os gestores das IES podem utilizar para evitar que a organização seja deficiente naquilo que é crítico para o sucesso e alcance de suas metas (Oliveira; Santos; Kalatzis, 2007).

É nesse contexto que Gonçalves, Colauto, Beuren (2005) realizaram sua pesquisa com o objetivo de identificar FCS em uma IES de Florianópolis, Santa Catarina. Os resultados mostram que a localização geográfica do campus, os recursos físicos, o horário das aulas foram destacados como mais importantes para alunos do curso de Administração. 
A pesquisa de Faria et al (2006) apresentou o grau de satisfação dos alunos do Curso de Ciências Contábeis de uma IES privada do Estado de São Paulo. A localização geográfica da instituição, composição do currículo, distribuição de carga horária das disciplinas, foram os FCS mais significativos, segundo a percepção de seus alunos.

Devido às muitas faces envolvidas no processo de Educação à Distância (EaD), várias pesquisas foram realizadas para identificar FCS em programas de EaD. Selim (2007) investigou os FCS que afetam a adoção de tecnologia de EaD por universidades na perspectiva dos estudantes, com destaque para a disponibilidade de laboratórios de informática para a execução das atividades, o acervo bibliográfico e disponibilidade para prestação de informações. Masrom, Zainon e Rahiman (2008) desenvolveram pesquisa sobre dois grupos de FCS: Tecnologia de Informação e Suporte da universidade. Entre os FCS mais importantes destacou-se a disponibilidade de laboratórios de computadores para os estudantes.

\section{Metodologia para a identificação dos FCS}

\subsection{Categorias de FCS}

Utilizou-se uma pesquisa bibliográfica para definir categorias de FCS e seus indicadores agrupando-os de acordo com características de cada categoria, como sugerido por Papp (2000). Foram definidas quatro categorias de FCS para a identificação de indicadores que influenciam no sucesso de um Curso de Ensino Superior Brasileiro independente da área ao qual está relacionado, seja ele presencial ou à distância. São elas: Instituição (19), Docente (18), Aluno (17) e Serviços de Apoio da Instituição (13).

Considerando que o meio em que o aluno está inserido faz parte do contexto educacional, com o FCS Instituição buscou-se avaliar a influência da instituição para o sucesso e aceitação dos cursos de graduação em Computação, enquanto que com o FCS Serviços de Apoio da Instituição, o foco da avaliação é no suporte oferecido aos alunos.

$\mathrm{Na}$ mesma perspectiva, dirigiu-se a pesquisa de modo a analisar a influência de dois atores essenciais, o aluno como agente ativo e participativo do processo da sua aprendizagem e o docente como agente na mediação entre o aluno e a busca por novos conhecimentos, justificando as categorias de FCS Docente e Aluno.

Os indicadores utilizados em cada categoria de FCS são mostrados na Tabela 1.

Tabela 1. FCS por indicadores.

\begin{tabular}{|c|c|c|}
\hline FCS & Indicadores & Fonte \\
\hline Instituição & $\begin{array}{l}\text { Q.14-Infraestrutura e instalações da universidade; Q.15-Percepção da qualidade dos } \\
\text { serviços prestados; Q.16-Imagem da universidade oferecida por alunos que já estão } \\
\text { cursando uma graduação; Q.17-Número e variedade de cursos oferecidos pela } \\
\text { universidade; Q.18-Compromisso da universidade com o serviço prestado; Q.19- } \\
\text { Marketing da universidade; Q.20-Geração, aceitação e implementação na } \\
\text { universidade de novas ideias, processos, produtos ou serviços; Q.21-Regulamentação } \\
\text { e adaptação de espaços para uso dos alunos, funcionários e comunidade; Q.22- } \\
\text { Comentários de satisfação ou insatisfação dos alunos atuais com o curso e com a } \\
\text { universidade; Q.23-Práticas administrativas da universidade; Q.24-Adoção de } \\
\text { ferramentas de EAD como apoio no processo de ensino e aprendizagem; Q.25- } \\
\text { Propensão da universidade à inovação; Q.26-Preocupação da universidade com seus } \\
\text { alunos; Q.27-Atmosfera positiva entre pessoas e ambientes na universidade; Q.28- } \\
\text { Compromisso da universidade com a comunidade e relaçães com sociedade e } \\
\text { natureza; Q.29-Segurança no campus da universidade; Q.30-Abertura da } \\
\text { universidade para a participação democrática; Q.31-Atendimento dos servidores e } \\
\text { funcionários da universidade; Q.32-Localização Geográfica do campus. }\end{array}$ & $\begin{array}{l}\text { Gonçalves, } \\
\text { Colauto, Beuren, } \\
\text { 2005; } \\
\text { Faria et al, 2006; } \\
\text { Duarte, Oliveira, } \\
\text { Braga, 2004; } \\
\text { Cheawjindakarn, } \\
\text { Suwannatthachote, } \\
\text { Theeraroungchaisri, } \\
\text { 2012. }\end{array}$ \\
\hline
\end{tabular}




\begin{tabular}{|c|c|c|}
\hline FCS & Indicadores & Fonte \\
\hline Docente & $\begin{array}{l}\text { Q.33-Qualificação do Corpo Docente; Q.34-Metodologia aplicada à realidade de } \\
\text { mercado; Q.35-Pontualidade; Q.36-Assiduidade; Q.37-Disponibilidade para } \\
\text { atendimento aos alunos fora do horário de aula; Q.38-Disponibilidade de meios } \\
\text { alternativos de comunicação com os alunos, como e-mail; Q.39-Resposta às dúvidas } \\
\text { dos alunos no menor tempo possível; Q.40-Utilização de linguagem clara e acessível } \\
\text { na explicação dos conteúdos, trabalhos e atividades; Q.41-Domínio atualizado dos } \\
\text { conteúdos das disciplinas sob sua responsabilidade; Q.42-Conhecimento das normas } \\
\text { da universidade; Q.43-Apresentação do plano de ensino; Q.44-Entusiasmo do } \\
\text { docente com o ensino; Q.45-Recursos tecnológicos utilizados pelo docente; Q.46- } \\
\text { Incentivo a utilização de ferramentas de EAD como apoio no processo de } \\
\text { aprendizagem; Q.47-Auxílio aos alunos no uso de ferramentas de EAD; Q.48- } \\
\text { Incentivo à interação entre alunos/alunos e docentes/alunos; Q.49-Estímulo aos } \\
\text { alunos para participação das aulas e das atividades; Q.50-Estímulo aos alunos para } \\
\text { busca de outras fontes de informação. }\end{array}$ & $\begin{array}{l}\text { Duarte, Oliveira, } \\
\text { Braga, 2004; } \\
\text { Gonçalves, } \\
\text { Colauto, Beuren, } \\
\text { 2005; } \\
\text { Selim, 2007; } \\
\text { Cheawjindakarn, } \\
\text { Suwannatthachote, } \\
\text { Theeraroungchaisri, } \\
2012 .\end{array}$ \\
\hline Aluno & $\begin{array}{l}\text { Q.51-Habilidade com o uso de computadores; Q.52-Experiência anterior com o uso } \\
\text { de computadores; Q.53-Percepção de ferramentas de EAD como apoio no processo } \\
\text { de ensino e aprendizagem; Q.54-Uso efetivo de ferramentas de EAD como auxílio no } \\
\text { processo de ensino e aprendizagem; Q.55-Percepção da interdisciplinaridade do } \\
\text { curso com outros cursos e/ou áreas de conhecimento; Q.56-Participação em grupos } \\
\text { de discussão sobre disciplinas ou temas inerentes ao curso; Q.57-Envolvimento com } \\
\text { o curso; Q.58-Disponibilidade de tempo para estudo fora do horário de aula; Q.59- } \\
\text { Busca novas fontes de informações; Q.60-Ser interativo com os docentes e com os } \\
\text { demais alunos; Q.61-Conhecimento do Projeto Pedagógico do Curso; Q.62- } \\
\text { Conhecimento das normas acadêmicas da universidade; Q.63-Estágio; Q.64- } \\
\text { Monitoria; Q.65-Participação em projetos de pesquisa, como atividades de iniciação } \\
\text { científica ou iniciação tecnológica; Q.66-Participação em projetos de extensão; Q.67- } \\
\text { Participação na Empresa Júnior. }\end{array}$ & $\begin{array}{l}\text { Duarte, Oliveira, } \\
\text { Braga, 2004; } \\
\text { Selim, 2007; } \\
\text { Cheawjindakarn, } \\
\text { Suwannatthachote, } \\
\text { Theeraroungchaisri, } \\
2012 .\end{array}$ \\
\hline $\begin{array}{c}\text { Serviços de } \\
\text { Apoio da } \\
\text { Instituição }\end{array}$ & $\begin{array}{l}\text { Q.68-Disponibilidade de equipamentos e laboratórios para o desenvolvimento do } \\
\text { curso; Q.69-Promoção e divulgação de atividades de extensão; Q.70-Promoção e } \\
\text { divulgação de eventos científicos; Q.71-Divulgação de informações gerais da } \\
\text { universidade para o aluno; Q.72-Atualização contínua do site da universidade; Q.73- } \\
\text { Serviços de internet disponíveis para uso dos alunos, como fonte de pesquisa } \\
\text { acadêmica e e-mail institucional; Q.74-Acervo disponível na biblioteca; Q.75- } \\
\text { Horário de funcionamento da biblioteca; Q.76-Atendimento e orientação dos setores } \\
\text { de apoio ao ensino (Biblioteca, Registro Escolar, Secretaria do Curso); Q.77-Adoção } \\
\text { de Ferramentas e Ambientes de EAD; Q.78-Disponibilização de suporte técnico para } \\
\text { auxílio no uso de ferramentas de EAD; Q.79-Adoção de cotas para impressão de } \\
\text { materiais didáticos; Q.80-Veiculação das informações nos setores da universidade. }\end{array}$ & $\begin{array}{l}\text { Duarte, Oliveira, } \\
\text { Braga, 2004; } \\
\text { Selim, 2007; } \\
\text { Masrom, Zainon e } \\
\text { Rahiman, 2008; } \\
\text { Cheawjindakarn, } \\
\text { Suwannatthachote, } \\
\text { Theeraroungchaisri, } \\
2012 .\end{array}$ \\
\hline
\end{tabular}

\subsection{Questionário}

A fim de identificar os FCS mais relevantes para cursos de graduação em Computação sob a visão dos alunos, foi elaborado um questionário estruturado contendo cinco seções, uma para cada categoria de FCS, além de uma seção contemplando questões demográficas. Logo, o questionário apresenta os 67 indicadores agrupados por categoria de FCS. Já as respostas foram organizadas em escala Likert (Likert, 1931) de cinco pontos, conforme Tabela 2. Ou seja, para cada item de resposta foi atribuído um número que reflete a direção da atitude do entrevistado em relação a cada indicador relacionado a um FCS (Malhotra, 2001). (p.e.: "Classifique o indicador Qualificação do Corpo Docente segundo o grau de relevância que ele assume para o FCS Docente").

Tabela 2. Alternativas de questionamento em escala Likert usados na pesquisa.

\begin{tabular}{|c|c|}
\hline Ponto da Escala Likert & Significado \\
\hline 5 & Concordo totalmente \\
\hline 4 & Concordo \\
\hline 3 & Indiferente \\
\hline 2 & Discordo \\
\hline 1 & Discordo totalmente \\
\hline
\end{tabular}


Nota: Quanto mais próximo de 5 a média estiver maior será a relevância do indicador e quanto mais próximo estiver de 1 menor será a relevância do indicador.

Para estimar a confiabilidade do questionário aplicado na pesquisa, foi utilizado o teste alpha de Cronbach (Cronbach, 1951). O coeficiente mede a correlação entre respostas em um questionário por meio da análise do perfil das respostas dadas pelos entrevistados. O coeficiente $\alpha$ é calculado a partir da variância dos itens individuais e da variância da soma dos itens de cada avaliador (Cronbach, 1951), sendo expresso pela equação Eq.01:

$$
\alpha=\left(\frac{k}{k-1}\right) *\left(1-\frac{\sum_{i=1}^{k} s_{i}^{2}}{s_{t}^{2}}\right)
$$

onde: $k$ corresponde ao número de itens do questionário; $s_{i}^{2}$ corresponde à variância de cada item; e $s_{t}^{2}$ corresponde à variância total do questionário, determinada como a soma de todas as variâncias.

Quando $\alpha \geq 0.9$ a confiabilidade do questionário é considerada excelente, segundo Cortina (1993). Para o questionário usado na pesquisa obteve-se $\alpha=0.975$, caracterizando um alto grau de confiabilidade.

\section{Apresentação e análise dos resultados}

A aplicação do questionário ocorreu nos períodos de 09 à 16 de dezembro de 2014 na UFRA e de 09 à 13 de março de 2015 na UFPA. Na UFRA foram entrevistados alunos dos cursos de Licenciatura em Computação e Sistemas de Informação, enquanto que na UFPA preencheram o questionário alunos dos cursos de Ciência da Computação, Engenharia da Computação e Sistemas de Informação. No total, 284 alunos participaram da pesquisa. O software utilizado para a obtenção das estatísticas apresentadas e do teste alpha de Cronbach foi o IBM SPSS Statistics Viewer. O perfil demográfico da pesquisa é mostrado na Tabela 3.

Tabela 3. Perfil demográfico e estatísticas descritivas dos alunos pesquisados.

\begin{tabular}{|c|c|c|}
\hline Item & Frequência & Porcentagem \\
\hline $\begin{array}{c}\text { Sexo: Masculino } \\
\text { Feminino }\end{array}$ & $\begin{array}{c}215 \\
69\end{array}$ & $\begin{array}{l}75,7 \\
24,3\end{array}$ \\
\hline $\begin{array}{l}\text { Raça: Branca ou Amarela } \\
\text { Preta ou Parda } \\
\text { Indígena }\end{array}$ & $\begin{array}{c}73 \\
210 \\
1\end{array}$ & $\begin{array}{c}25,71 \\
73,94 \\
0,35\end{array}$ \\
\hline $\begin{array}{r}\text { Renda bruta familiar (em salários mínimos): Até } 1 \\
\text { De } 1 \text { a } 3 \\
\text { Mais de } 3\end{array}$ & $\begin{array}{c}67 \\
135 \\
82\end{array}$ & $\begin{array}{l}23,59 \\
47,54 \\
28,87\end{array}$ \\
\hline $\begin{array}{c}\text { Tipo de escola que estudou o Ensino Médio: Pública } \\
\text { Privada } \\
\text { Pública e Privada }\end{array}$ & $\begin{array}{c}170 \\
96 \\
18 \\
\end{array}$ & $\begin{array}{c}59,86 \\
33,8 \\
6,34 \\
\end{array}$ \\
\hline $\begin{array}{c}\text { Vive em: Área Urbana } \\
\text { Área Rural }\end{array}$ & $\begin{array}{c}280 \\
4\end{array}$ & $\begin{array}{c}98,6 \\
1,4\end{array}$ \\
\hline $\begin{array}{l}\text { Já utilizava o computador antes de iniciar o curso de graduação: Não } \\
\text { Sim } \\
\text { Há menos de } 1 \text { ano } \\
\text { De } 1 \text { à } 4 \text { anos } \\
\text { De } 4 \text { à } 8 \text { anos } \\
\text { Há mais de } 8 \text { anos }\end{array}$ & $\begin{array}{c}31 \\
253 \\
4 \\
48 \\
80 \\
121\end{array}$ & $\begin{array}{c}10,92 \\
89,08 \\
1,58 \\
18,97 \\
31,62 \\
47,83\end{array}$ \\
\hline $\begin{array}{c}\text { Possuem computador próprio: Não } \\
\text { Sim }\end{array}$ & $\begin{array}{c}29 \\
255\end{array}$ & $\begin{array}{l}10,24 \\
89,76\end{array}$ \\
\hline $\begin{array}{l}\text { Possuem acesso à Internet em casa: Não } \\
\text { Sim }\end{array}$ & $\begin{array}{c}37 \\
247\end{array}$ & $\begin{array}{l}13,03 \\
86,97\end{array}$ \\
\hline
\end{tabular}


Analisando as médias obtidas para cada categoria de FCS, nota-se que o FCS Docente foi apontado como o de maior relevância $(3,86)$ segundo a perspectiva dos alunos. O FCS Aluno $(3,83)$ foi considerado o segundo fator de maior relevância, seguido do FCS Serviços de Apoio da Instituição $(3,7)$ e do FCS Instituição $(3,59)$. Estes dados são mostrados na Figura 1.

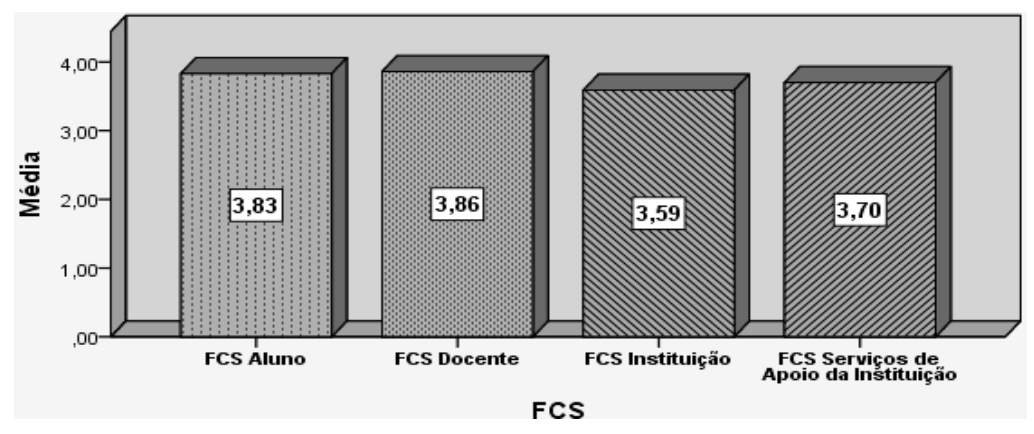

Figura 1. Média por categoria de FCS.

A Tabela 4 apresenta o resultado da pesquisa dos indicadores agrupados por FCS.

Tabela 4. Estatísticas para os indicadores dos FCS.

\begin{tabular}{|c|c|c|c|}
\hline FCS & Indicadores & Média & Desvio padrão \\
\hline \multirow{19}{*}{ 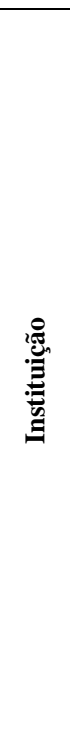 } & Q14 & 3,77 & 1,036 \\
\hline & Q15 & 3,59 & 1,000 \\
\hline & Q16 & 3,64 & ,833 \\
\hline & Q17 & 3,53 & 1,013 \\
\hline & Q18 & 3,74 & 1,026 \\
\hline & Q19 & 3,05 & 1,024 \\
\hline & Q20 & 3,72 & 1,036 \\
\hline & Q21 & 3,58 & 1,108 \\
\hline & Q22 & 3,54 & ,945 \\
\hline & Q23 & 3,62 & ,971 \\
\hline & Q24 & 3,63 & 1,016 \\
\hline & Q25 & 3,85 & 1,039 \\
\hline & Q26 & 3,64 & 1,192 \\
\hline & Q27 & 3,75 & ,973 \\
\hline & Q28 & 3,77 & ,871 \\
\hline & Q29 & 3,55 & 1,266 \\
\hline & Q30 & 3,62 & 1,057 \\
\hline & Q31 & 3,45 & 1,101 \\
\hline & Q32 & 3,18 & 1,222 \\
\hline \multirow{17}{*}{$\stackrel{\stackrel{\Xi}{\Xi}}{z}$} & Q51 & 4,13 & ,694 \\
\hline & Q52 & 3,87 & ,828 \\
\hline & Q53 & 3,61 & 1,020 \\
\hline & Q54 & 3,51 & 1,033 \\
\hline & Q55 & 3,82 & 1,066 \\
\hline & Q56 & 3,60 & 1,055 \\
\hline & Q57 & 4,19 & ,858 \\
\hline & Q58 & 3,88 & 1,034 \\
\hline & Q59 & 4,18 & ,836 \\
\hline & Q60 & 4,00 & ,795 \\
\hline & Q61 & 3,68 & ,946 \\
\hline & Q62 & 3,52 & 1,044 \\
\hline & Q63 & 4,16 & ,949 \\
\hline & Q64 & 3,79 & 1,083 \\
\hline & Q65 & 3,98 & 1,103 \\
\hline & Q66 & 3,84 & 1,093 \\
\hline & Q67 & 3,40 & 1,244 \\
\hline
\end{tabular}

\begin{tabular}{|l|l|r|r|}
\hline FCS & Indicadores & Média & Desvio Padrão \\
\hline \multirow{7}{*}{} & Q33 & $\mathbf{4 , 3 4}$ & $\mathbf{, 7 6 7}$ \\
\cline { 2 - 4 } & Q34 & 3,85 & 1,032 \\
\cline { 2 - 4 } & Q35 & 3,80 &, 927 \\
\cline { 2 - 4 } & Q36 & 3,83 &, 943 \\
\cline { 2 - 4 } & Q37 & 3,73 & 1,007 \\
\cline { 2 - 4 } & Q38 & $\mathbf{4 , 0 6}$ & $\mathbf{9 7 1}$ \\
\cline { 2 - 4 } & Q39 & 3,85 &, 939 \\
\cline { 2 - 4 } & Q40 & 3,96 &, 961 \\
\cline { 2 - 4 } & Q41 & $\mathbf{4 , 0 1}$ &, $\mathbf{9 4 9}$ \\
\cline { 2 - 4 } & Q42 & 3,75 &, 934 \\
\cline { 2 - 4 } & Q43 & 3,94 &, 907 \\
\cline { 2 - 4 } & Q44 & 3,83 &, 924 \\
\cline { 2 - 4 } & Q45 & 3,91 &, 917 \\
\cline { 2 - 4 } & Q46 & 3,57 & 1,058 \\
\cline { 2 - 4 } & Q47 & 3,40 & 1,054 \\
\cline { 2 - 4 } & Q48 & 3,75 &, 946 \\
\cline { 2 - 4 } & Q49 & 3,86 &, 861 \\
\cline { 2 - 4 } & Q50 & 3,97 &, 894 \\
\hline
\end{tabular}

\begin{tabular}{|c|c|c|c|}
\hline \multirow{13}{*}{ 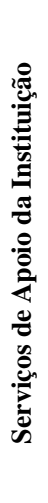 } & Q68 & 3,98 & 1,178 \\
\hline & Q69 & 3,69 & 1,130 \\
\hline & Q70 & 3,86 & 1,052 \\
\hline & Q71 & 3,77 & 1,007 \\
\hline & Q72 & 3,84 & 1,104 \\
\hline & Q73 & 3,99 & 1,037 \\
\hline & Q74 & 4,09 & 1,030 \\
\hline & Q75 & 3,73 & 1,190 \\
\hline & Q76 & 3,70 & 1,141 \\
\hline & Q77 & 3,49 & 1,030 \\
\hline & Q78 & 3,44 & 1,053 \\
\hline & Q79 & 3,21 & 1,290 \\
\hline & Q80 & 3,43 & 1,235 \\
\hline
\end{tabular}


Para os indicadores da categoria FCS Instituição, o destaque é para Q.25Propensão da universidade à inovação. A inovação promove o desenvolvimento econômico e social de forma sustentável (Lopes, 2012) e a propensão à inovação das universidades pesquisadas tendem a trazer benefícios para a Região Amazônica por meio da produção e divulgação do conhecimento científico e tecnológico, segundo os entrevistados. A indicação de Q.14-Infraestrutura e instalações da universidade mostra a relevância dada à necessidade de manutenção de boa infraestrutura e instalações refletindo na satisfação do aluno com o curso e com a universidade. A observância de Q.28-Compromisso da universidade com a comunidade e relações com sociedade e natureza traduz que o compromisso social das universidades com suas ações básicas de pesquisa, ensino e extensão são confirmadas pelos alunos como reflexo de excelência nos cursos, principalmente no tocante da formação de um profissional cidadão relacionado com a apropriação e produção do conhecimento científico, comprometido com a realidade social da região para atuar com desenvoltura na problemática regional. O indicador Q.27-Atmosfera positiva entre pessoas e ambientes na universidade mostra que o ambiente favorável ao bom convívio entre as pessoas na universidade também é relevante. Por sua vez, Q.18-Compromisso da universidade com o serviço prestado mostra que além do papel formador, os alunos entendem que a função social desempenhada pelas universidades permite aproximar a comunidade do que é produzido e aprendido no ambiente acadêmico. Este indicador pode colaborar para a disseminação do conhecimento e fornecer subsídios para que sejam elaboradas políticas públicas de Educação, Saúde, Tecnologia que possam minimizar as desigualdades e promover o crescimento sustentável da Amazônia.

Entre os indicadores do FCS Docente, observa-se que Q.33-Qualificação do Corpo Docente é visto como o de maior relevância na categoria, destacando a importância da qualificação do docente para a formação acadêmica dos alunos. Este indicador também obteve destaque nas pesquisas de Gonçalves, Colauto, Beuren (2005) e Faria et al (2006), embora com menor relevância. Q.38-Disponibilidade de meios alternativos de comunicação com os alunos, tal qual a pesquisa de Cheawjindakarn, Suwannatthachote e Theeraroungchaisri (2012), é percebido em nessa pesquisa como o segundo indicador de maior importância evidenciando a necessidade dos docentes manterem um meio secundário a disposição dos alunos para comunicação de assuntos com foco acadêmico favorecendo a comunicação entre alunos e docentes. A indicação de Q.41-Domínio atualizado dos conteúdos das disciplinas sob sua responsabilidade confirma a necessidade do docente em se manter sempre atualizado com o conteúdo das disciplinas pertencentes ao currículo dos cursos possibilitando o acompanhamento do dinamismo do mercado computacional e ainda contribuindo com avanço científico e tecnológico em especial da Região Amazônica.

$\mathrm{Na}$ categoria FCS Aluno, Q.57-Envolvimento com o curso foi considerado o mais importante, demonstrando que quanto mais o aluno tem interesse por um determinado assunto, disciplina ou curso mais estará propenso ao sucesso. Semelhante à pesquisa de Cheawjindakarn, Suwannatthachote e Theeraroungchaisri (2012), o indicador Q.59-Busca novas fontes de informações também obteve destaque. Nota-se que a pró-atividade dos alunos em buscar novas fontes de informação sobre temas, conteúdos e disciplinas do curso tem um impacto favorável na construção do conhecimento podendo ser um diferencial para o seu sucesso no curso bem como para o sucesso do próprio curso. Por sua vez, por intermédio de Q.63-Estágio os alunos entendem ser possível participar de forma mais consistente dentro do mercado de 
trabalho, destacando suas habilidades e competências em selecionar, gerenciar e desenvolver soluções de software e hardware que acelerar o desenvolvimento tecnológico na Amazônia. Este indicador também teve destaque na pesquisa de Duarte, Oliveira e Braga (2004). Ainda em 2006, foi considerado que o estudante que não possui conhecimento digital e acesso ao computador está limitado em suas oportunidades de aprendizado no Ensino Superior (Cafezeiro, 2006), o que foi percebido pelos alunos entrevistados devido à relevância dada à Q.51-Habilidade com o uso de computadores.

Para os indicadores da categoria FCS Serviços de Apoio da Instituição, Q.74Acervo disponível na biblioteca obteve maior relevância, mostrando a valorização dos estudantes pesquisa acadêmica e da leitura como forma de obtenção e valorização do conhecimento atingido, semelhante ao resultado encontrado por Selim (2007). Já o indicador Q.73-Serviços de internet disponíveis para uso dos alunos, destacado também na pesquisa de Cheawjindakarn, Suwannatthachote e Theeraroungchaisri (2012), tem sua importância como mecanismo facilitador de busca por outras fontes de informação para agregar valor ao conhecimento adquirido pelos alunos nos cursos. O indicador Q.68-Disponibilidade de equipamentos e laboratórios para o desenvolvimento foi também destacado na pesquisa de Duarte, Oliveira e Braga (2004), Selim (2007) e Masrom, Zainon e Rahiman (2008). Este indicador traduz a preocupação dos alunos com a disponibilidade de equipamentos que permitam melhor interação das disciplinas com a parte prática do curso.

Entre os indicadores com menor influência no sucesso dos cursos de graduação em Computação, destacam-se: Q.19-Marketing da Universidade (3,05) pertencente à categoria do FCS Instituição; Q.79-Adoção de cotas para impressão de materiais didático $(3,21)$ pertencente à categoria do FCS Serviços de Apoio da Instituição; Q.47Auxílio aos alunos no uso de ferramentas de $\operatorname{EAD}(3,4)$ pertencente à categoria do FCS Docente; e Q.67-Participação na Empresa Júnior $(3,4)$ pertencente à categoria do FCS Aluno.

A identificação dos fatores críticos de sucesso na percepção dos estudantes de graduação da área de Computação permite alinhar as estratégias da instituição, para que o egresso possa se tornar um profissional competente, ativo, empreendedor, ético e consciente do seu papel enquanto cidadão e conhecedor da importância da contribuição no avanço científico e tecnológico do país, e em especial da Região Amazônica.

\section{Considerações finais}

O artigo apresentou dados de uma pesquisa realizada com 284 alunos dos cursos de Ciência da Computação, Engenharia da Computação, Licenciatura em Computação e Sistemas de Informação da Universidade Federal Rural da Amazônia e da Universidade Federal do Pará, ambas situadas na Região Amazônica, entre 09 a 16 de dezembro de 2014 e 09 a 13 de março de 2015, com o objetivo de identificar fatores críticos de sucesso para esses cursos, segundo a ótica de seus alunos.

Para a pesquisa agrupou-se os fatores críticos em quatro categorias: Instituição, Docente, Aluno, Serviços de Apoio da Instituição. Cada categoria é composta por vários indicadores propostos em estudos de diversos pesquisadores da área.

Utilizou-se questionário com escala Likert de cinco pontos para produzir descrições quantitativas dos indicadores e assim, dos FCS. Os resultados foram 
determinados pela análise estatística dos itens Likert em cada categoria dos FCS. A confiabilidade do questionário foi estimada por meio do teste de alpha Cronbach que apresentou grau de confiabilidade de 0,975 .

Para os cursos de graduação em Computação obtiveram destaque os FCS Docente $(3,86)$, Aluno $(3,83)$, Serviços de Apoio da Instituição $(3,7)$ e Instituição $(3,59)$, nessa ordem de relevância.

Entre todos os indicadores analisados nas quatro categorias de FCS o indicador Q.33-Qualificação do Corpo Docente, pertencente à categoria do FCS Docente, foi o que apresentou maior média, 4,34. A qualificação do corpo docente também foi identificada como FCS nas pesquisas de Gonçalves, Colauto, Beuren (2005) e Faria et al (2006) realizadas nas Regiões Sul e Sudeste do Brasil, ainda que em menor nível de relevância. De acordo com os resultados encontrados, assume-se, então, que os docentes exercem um papel essencial no processo de obtenção de sucesso de cursos de graduação em Computação ao serem apontados como responsáveis pelo fluxo de produção, de disseminação do conhecimento e desenvolvimento científico e tecnológico da Região Amazônica.

A identificação, análise e divulgação dos FCS na percepção dos estudantes são importantes para que os gestores das instituições possam identificar pontos críticos para melhorar a gestão institucional, estratégica e operacional em face ao cumprimento dos objetivos propostos pelos cursos de graduação em Computação e às perspectivas dos alunos. A ideia é que essa estratégia minimize as desigualdades e propicie a produção de conhecimentos científicos e tecnológicos capazes de melhorar a qualidade de vida da população Amazônica.

Como trabalhos futuros, sugere-se que sejam utilizadas outras abordagens para validar e analisar a relação entre os indicadores, como o Modelo de Confirmação de Fatores proposto por Selim (2007), e que seja realizada uma avaliação do impacto dos FCS destacados nesta pesquisa, como em minimizar a evasão dos cursos, por exemplo.

\section{Referências}

Cafezeiro, I. (2006) "Educação, Informática e Responsabilidade Social: A Contribuição da Universidade Pública" XVII Simpósio Brasileiro de Informática na Educação SBIE, Brasília.

Cheawjindakarn,B.; Suwannatthachote,P.; Theeraroungchaisri, A. (2012) "Critical Success Factors for Online Distance Learning in Higher Education: A Review of the Literature". Creative Education, v. 3, Supplement, p. 61-66.

Cortina, J. (1993) "What is coefficient alpha? An examination of theory and applications" Journal of Applied Psychology. http://www.cb.wsu.edu/ dsprott/teaching/StGallen/Readings/Cortina_JAP_93.pdf, Maio, 2015.

Cronbach, J. (1951) "Coefficient alpha and the internal structure of tests" v. 16. n. 3, p. 297-334, Psychometrika.

Duarte, M.; Oliveira, A.; Braga, J. (2004) "Determinação semi-automática de fatores críticos de sucesso para a área acadêmica". Revista Informática Pública, v. 6, n. 1, p. 53-76. 
Faria, A.; Come, E.; Poli, J.; Felipe, Y. (2006) "O Grau de satisfação dos alunos do curso de Ciências Contábeis: busca e sustentação da vantagem competitiva de uma IES privada”. Revista Enfoque Reflexão Contábil, Paraná, v. 21, n. 1, p. 25-36.

Gonçalves, C.; Colauto, R.; Beuren, I. (2005) "Identificação dos Fatores Críticos de Sucesso em Instituição de Ensino Superior" Anais..., IV Colóquio Internacional Sobre Gestão Universitária na América Do Sul, Mar Del Plata.

Júnior, A.; Polizel, C.; Silva; P. (2012) "Fatores Críticos de Sucesso para a gestão do conhecimento em uma instituição de educação superior privada" Revista Brasileira de Gestão de Negócios, São Paulo, v. 14, n. 42, p.102-122.

Likert, R. (1931) "A technique for the measurement of attitudes. Archives of Psychology" New York: Columbia University Press.

Lopes, V. (2012) "A inserção da Universidade Federal do Sergipe (UFS) no processo de inovação e desenvolvimento local: Intenção e prática" Doutorado em Engenharia de Produção. Universidade Federal do Pernambuco: Recife, 2012

Masrom, M.; Zainon, O.; Rahiman, R. (2008) "Critical Success in E-learning: An Examination of Technological and Institutional Support Factors" International Joint Conference on e-Commerce, e-Administration, e-Society, and e-Education, Thailand.

Malhotra, N. (2001) Pesquisa de marketing: uma orientação aplicada (3a ed.). Porto Alegre: Bookman.

Nunes, D. (2012) "Educação Superior em Computação - Estatísticas 2012", http://www.sbc.org.br/index.php?option=com_jdownloads\&Itemid=195\&task=view. download\&catid=39\&cid=567, Abril, 2014 .

Oliveira, S.; Santos, E.; Kalatizis, A. (2007) "Suporte metodológico para aperfeiçoamento de planejamento de EAD utilizando estilos de aprendizagem, inteligências múltiplas e competências requeridas: um estudo multi-casos nos cursos de Administração" EnEPQ.

Papp, R. (2000) "Critical success factors for distance learning" Americas Conference on Information Systems, Long Beach, CA, USA.

Retamal, D. (2009) "A gestão de cursos de graduação via internet: uma visão a partir dos fatores críticos de sucesso". Universidade Federal do Rio Grande do Sul, http://www.lume.ufrgs.br/bitstream/handle/10183/18252/000727582.pdf?sequence=1 , Abril/2014.

Rockart, J. (1979) Chief executives define their own data needs. Harvard Business Review, v.57, n.2, p.81-93.

Selim, H. (2007) "Critical Success factors for e-learning acceptance: Confirmatory factor models", Computers \& Education, n. 49. 